\begin{tabular}{|c|l|}
\hline Title & Selective monofluorination of diols using DFMBA \\
\hline Author(s) & Yoneda, A tsushi; Fukuhara, Tsuy oshi; Hara, Shoji \\
\hline Citation & $\begin{array}{l}\text { Chemical Communications, 2005(28), 3589-3590 } \\
\text { https://doi.org/10.1039/6502471d }\end{array}$ \\
\hline Issue Date & 2005 \\
\hline Doc URL & http://hdl.handle.net/2115/36649 \\
\hline Rights & Chem. Commun., 2005, 3589-3590- Reproduced by permission of The Royal Society of Chemistry (RSC) \\
\hline Type & article (author version) \\
\hline File Information & hara2.pdf \\
\hline
\end{tabular}

Instructions for use 


\title{
Selective monofluorination of diols using DFMBA
}

\author{
Atsushi Yoneda, Tsuyoshi Fukuhara and Shoji Hara* \\ Receipt/Acceptance Data [DO NOT ALTER/DELETE THIS TEXT] \\ Publication data [DO NOT ALTER/DELETE THIS TEXT] \\ DOI: 10.1039/b000000x [DO NOT ALTER/DELETE THIS TEXT]
}

\begin{abstract}
Selective monofluorination of 1,2- or 1,3-diols was achieved by the reaction with DFMBA. The method is applicable for the synthesis of optically active fluorohydrin derivatives.
\end{abstract}

Selective monofluorination of 1,2- or 1,3-diols is useful methodology for the synthesis of fluorinated sugars, ${ }^{1}$ nucleocides, ${ }^{2}$ or optically active fluorohydrins. ${ }^{3}$ However, it is difficult to selectively convert one hydroxy group of the diols to fluoride and leave another one unchanged, because prevention of the second deoxyfluorination reaction is usually difficult. Moreover, when 1,2- or 1,3-diols were treated with diethylaminosulfur trifluoride (DAST), the most typical deoxyfluorination reagents, or deoxyfluor ${ }^{\mathrm{TM}}$, its analog, side reactions such as rearangement ${ }^{4}$ or cyclic sulfonate formation ${ }^{5}$ competitively took place, and the expected fluorination products could not be obtained in good yields. Recently, we reported that primary and anomeric hydroxy groups in sugars can be selectively converted to fluoride by $N, N$-diethyl$\alpha, \alpha$-difluoro(m-methylbenzyl)amine (DFMBA). ${ }^{6}$ We wish to report here the selective monofluorination of diols using DFMBA and its application for the synthesis of optically active fluorohydrine derivatives.

When ethylene glycohol (1a) was subjected to the reaction with 2.4 eq of DFMBA in heptane at $98{ }^{\circ} \mathrm{C}$ for $1 \mathrm{~h}, m$-methylbenzoyl ester of 2-fluoroethanol (2a) was obtained in $79 \%$ yield. Only one hydroxy group of 1a was fluorinated and the other hydroxy group was esterificated by DFMBA. When the reaction was carried out under irradiation of microwave, the reaction was completed in 10 min and 2a was obtained in $73 \%$ yield.

Under similar conditions, various 1,2-diols (1a,c,d) and 1,3-diols $(\mathbf{1 b}, \mathbf{e}, \mathbf{f})$ could be converted to the corresponding fluorohydrine derivatives in good yields as shown in Table 1 . When an unsymmetrical diol (1f) was used, a mixture of two regioisomers was obtained nonselectively. On the other hand, the reaction of 1,12-dodecanediol (19), in which the hydroxy groups are separated by many methylene groups, gave a difluorinated product in good yield.

Any special care is not necessary to terminate the reaction at monofluorinated step. Therefore, the reaction seems to be proceeding through a cyclic intermediate, and after monofluoriantion reaction, the remained hydroxy group was changed to the ester group which is inert to DFMBA (Scheme 1).

When an optically active $(2 S, 4 S)$-2,4-pentandiol (1h) was subjected to the reaction with DFMBA, a monofluorinated product (2h) was obtained in $75 \%$ yield with high diastereoselectivity. In order to examine the stereochemistry of the reaction, $\mathbf{1 h}$ was converted to the monomesylate $\mathbf{3}$. Monofluorination of $\mathbf{3}$ was carried out with

† Electronic Supplementary Information (ESI) available: [details of any supplementary information available should be included here]. See http://www.rsc.org/suppdata/xx/b0/b000000x/

*xxxx@aaa.bbb.ccc
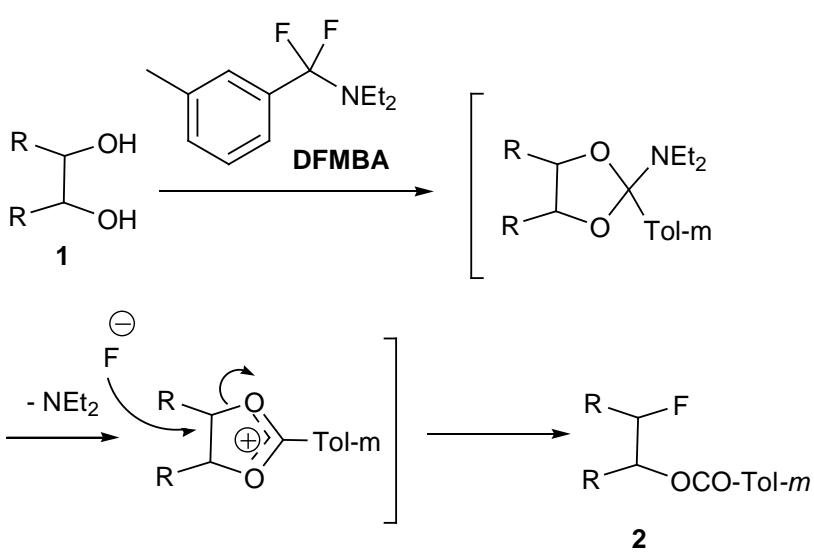

Scheme 1

the inversion of stereochemistry by TBAF $5 \mathrm{H}_{2} \mathrm{O}^{7}$ to give $(2 S, 4 R)$ 4-fluoro-2-pentanol $m$-methylbenzoyl ester. As its ${ }^{1} \mathrm{H}$ and ${ }^{19} \mathrm{~F}$ NMR spectra, and optical rotation ${ }^{8}$ coincided with those of $\mathbf{2 h}$, fluorination of alcohols by DFMBA was found to proceed with inversion of stereochemistry. From $(2 R, 4 R)-\mathbf{1 h},(2 R, 4 S)-\mathbf{2 h}$ was obtained selectively (Scheme 2).
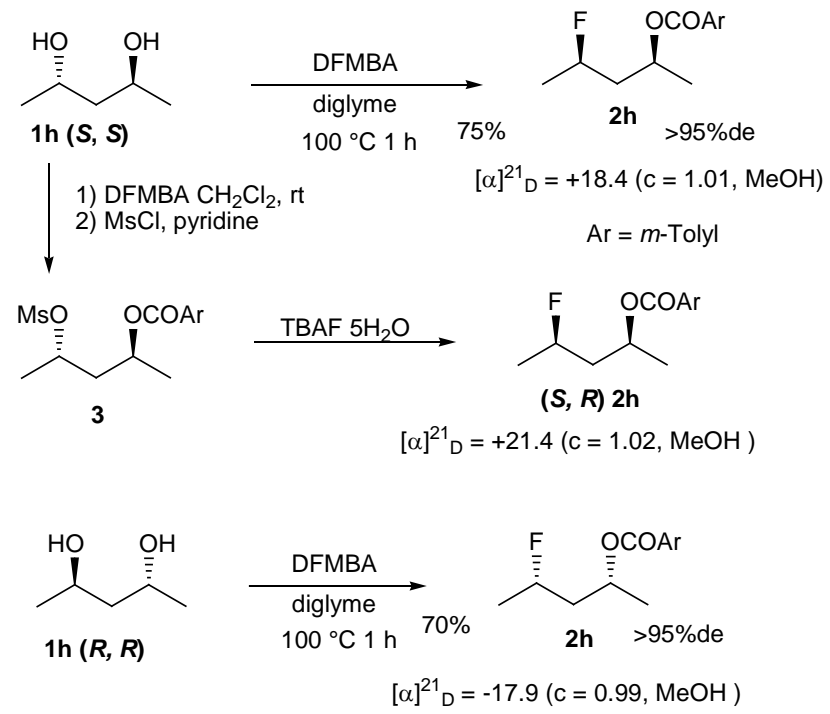

Scheme 2

In a similar manner, optically active fluorohydrine derivatives could be obtained from commercially available optically acitive 2,3-pentanediol (1i), 1,2-diphenylethanediol (1j) with high diastereoselectivity. In nature, many compounds such as sugars have optically active diol functions. When a manitol derivative 
(1k) was subjected to the reaction with DFMBA, a monofluorinated product (2k) was obtained with high diastereoselectivity.

Table 1 Reaction of diols with DFMBA

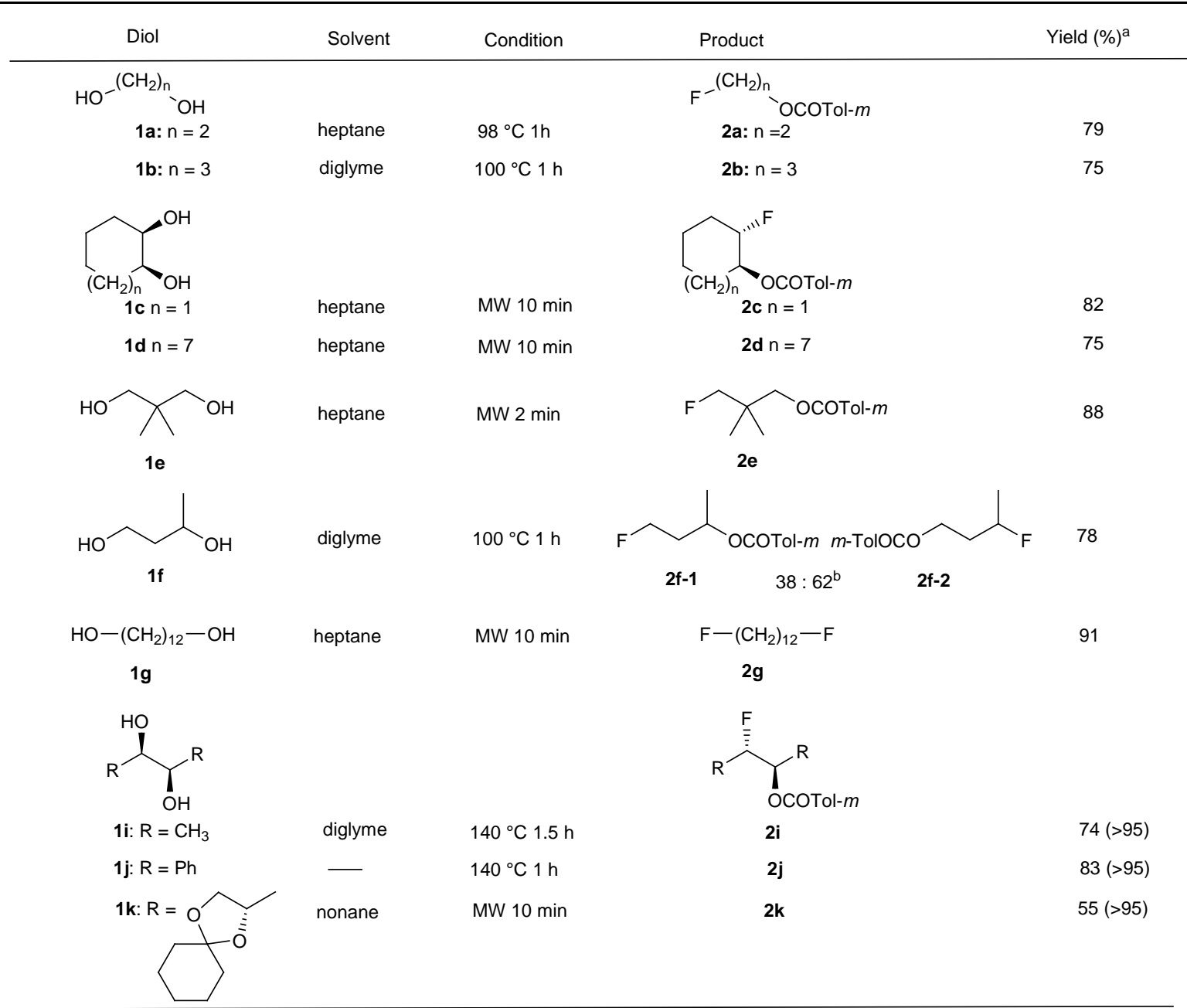

${ }^{a}$ Isolated yields based on diols used. In parentheses, diastereoselectivities. ${ }^{\mathrm{b}}$ Determined by ${ }^{19} \mathrm{FNMR}$.

Atushi Yoneda, Tsuyoshi Fukuhara and Shoji Hara*

Division of Molecular Chemistry, Graduate School of Engineering, Hokkaido University, Sapporo 060-8628, Japan

\section{Notes and references}

$\dagger$ General procedure of mono-fluorination of diols using DFMBA: A mixture of diol (1 mmol), DFMBA (2.2 mmol), and solvent $(1 \mathrm{ml})$ in a Teflon PFA $^{\text {TM }}$ vessel was heated by microwave ${ }^{6}$ or oil bath at the temperatre and for the time shown in Table 1 . After the reaction, the mixture was poured into aq $\mathrm{NaHCO}_{3}$ and extracted with ether three times. The combined organic phases were dried over $\mathrm{MgSO}_{4}$, concentrated under reduced pressure, and purified by column chlomatography (silica gel/ hexane-ether) to give monofluoride 2 .

1 K. Dax, M. Albert, J. Ortner, B. J. Paul, Carbohydr. Res., 2000, 327, 47.

2 K. W. Pankiewicz, Carbohydr. Res., 2000, 327, 87.
3 G. Haufe, J. Fluorine Chem., 2004, 125, 875.

4 C. Ye, J. M. Shreeve, J. Fluorine Chem., 2004, 125, 1869.

5 D. F. Shellhamer, D. T. Anstine, K. M. Gallego, B. R. Ganesh, A. A. Hanson, K. A. Hanson, R. D. Henderson, J. M. Prince, V. L. Heasley, J. Chem. Soc., Perkin Trans. 2., 1995, 861.

6 S. Kobayashi, A. Yoneda, T. Fukuhara, S. Hara, Tetrahedron Lett., 2004, 45, 1287; S. Kobayashi, A. Yoneda, T. Fukuhara, S. Hara, Tetrahedron, 2004, 60, 6923.

7 D. Albanese, D. Landini, M. Penso, J. Org. Chem., 1998, 63, 9587.

8 (2R, 4S)-2h, prepared by the reaction with $\mathrm{TBAF}-5 \mathrm{H}_{2} \mathrm{O}$, was contaminated by about $10 \%$ of olefinic by-products which were difficult to separate from $\mathbf{2 h}$, and it must be the reason why it had a larger value in optical rotation than that of $2 \mathbf{h}$ prepared from $\mathbf{1 h}$ and DFMBA. 